\title{
PUBLICACIÓN CIENTÍFICA Y CARACTERÍSTICAS DE ASESORES DE TESIS DE UNA ESCUELA DE PSICOLOGÍA PERUANA
}

\author{
Oscar J. Mamani Benito ${ }^{1 \mathrm{a}}$ \\ Escuela Profesional de Psicología, Universidad Peruana Unión ${ }^{1}$
}

Orcid ID: https://orcid.org/0000-0002-9818-2601 ${ }^{1}$

\section{Recibido: 28 de junio de 2018}

Aceptado: 25 de noviembre de 2018

\section{Resumen}

El objetivo de la presente fue describir las características de los asesores de tesis de pregrado en una escuela de psicología y analizar su producción científica. La metodología implica un estudio de tipo descriptivo-bibliométrico, donde la población estuvo constituida por 37 docentes asesores de 149 trabajos de pregrado aprobados en una universidad privada del Perú, los mismos que fueron ubicados en el repositorio digital de dicha institución y aprobados en el periodo 2014 - 2017. Los principales resultados indican que hubo una mayor participación de asesores mujeres $(70.3 \%)$ que varones $(29.7 \%$ ), además, el $64.9 \%$ evidencia tener solo título profesional, el $21.6 \%$ grado de magister y tan solo un $13.5 \%$ grado de doctor, así mismo, solo el $27 \%$ evidencia publicaciones científicas en Google Académico, mientras que el $73 \%$ no publicó alguna vez en su vida; finalmente, se halló una producción científica de 37 artículos (33 originales, 2 cartas al editor y 2 editoriales) correspondientes a 10 docentes asesores, en revistas nacionales e internacionales. Se concluye que los asesores de tesis de pregrado en psicología tienen una preocupante y baja producción científica.

Palabras clave: Asesor de tesis; producción científica; tesis; artículo científico

\section{SCIENTIFIC PUBLICATION AND CHARACTERISTICS OF THE THESIS ADVISERS OF A PERUVIAN PSYCHOLOGY SCHOOL}

\begin{abstract}

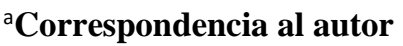

E-mail: oscar.mb@upeu.edu.pe
\end{abstract}


The objective of this study was to describe the characteristics of undergraduate thesis advisors in a psychology school and analyze their scientific production. The methodology involves a descriptive-bibliometric study, where the study population was 37 teacher advisors of 149 undergraduate projects approved at a private university in Peru. These were placed in the institution's repository and approved in the period from 2014-2017. The main results indicate that there was greater participation of women advisers (70.3\%) than men (29.7\%), in addition, $64.9 \%$ had only a professional title (undergraduate degree), $21.6 \%$ a master's degree and only $13.5 \%$ a doctorate degree. Likewise, only $27 \%$ evidence scientific publications in Google Scholar, while $73 \%$ did not publish sometime in their life; finally, we found a scientific production of 37 articles published (33 originals, 2 letters to the editor and 2 editorials) corresponding to 10 advisory teachers, in national and international journals. In conclusion, the undergraduate thesis advisors in psychology have a worrying and low level scientific production.

Keywords: Thesis advisor; scientific production; thesis; scientific article

\section{Introducción}

La tesis viene a representar un documento académico que es producto de un proceso de investigación científica validado según normas o políticas de investigación de cada universidad. En el Perú, luego de casi 25 años, periodo en el que su realización obligatoria para optar el título profesional en el pregrado fuera relegada a ser una de tres alternativas establecidas según el decreto legislativo N 739 en el año 1991 (San Martin \& Garcia, 2006), nuevamente se la reinstauró como indispensable en el año 2014, con la finalidad de impulsar nuevamente la producción científica estudiantil, así, el estado peruano mediante un nuevo decreto legislativo $\mathrm{N}^{\circ} 30220$ nuevamente consigna devolver el carácter obligatorio de su realización para la titulación, esto, debido a circunstancias negativas y poco promisorias para el país, como es el caso del año 2015 donde Perú fue considerado como el país con la más baja proyección de producción científica en Latinoamérica (Alarco, Changllio-Calle, \& Cahuana-Salazar, 2017); al respecto, este hecho también fue corroborado por diversos estudios nacionales reportados en la literatura científica donde también se afirma que la publicación científica sobre todo de las tesis es baja y preocupante (Atamari-Anahui, Roque-Roque, Robles-Mendoza, Nina, \& Falcón-Huancahuiri, 2015a; CastroRodríguez, 2018; Huaraca, Apaza, Mejia, 2017; Miyahira, 2015).

Este nuevo enfoque consignado en la nueva Ley universitaria (Ministerio de Educacion, 2014) implica no solo la realización obligatoria de una tesis para el título profesional sino también la elaboración de un trabajo de investigación para obtener el grado de bachiller. Con estos cambios, claramente se busca devolver la importancia de generar nuevo conocimiento a partir de los trabajos de grado desde las universidades, los cuales además, se espera que generen impacto social y calidad 
metodológica que empodere las posibilidades de publicación en revistas indexadas de la especialidad. Es por ello, que en la actualidad existe una nueva forma de comprender la realización de una tesis, pues tal como lo afirma León (2016) significa tres cosas: un aporte al conocimiento científico que hace la universidad al país a través del trabajo del alumno, una oportunidad de formación de nuevos investigadores y finalmente como un medio de certificación de las competencias que la universidad confiere.

Por otro lado, al enfocar el análisis solo en el primer significado que implica un aporte al conocimiento científico del país, es necesario comprender que esta contribución puede hacerse de dos maneras: los repositorios digitales donde se suben los trabajos en extenso y la publicación de un artículo científico en una revista indexada. Al respecto, se tiene que reconocer que este último, es el que mayor alcance y utilidad tiene en la comunidad científica, pues para la publicación en revistas de especialidad existen filtros como la revisión por pares a cargo de expertos, quienes evalúan con mayor precisión la fidelidad, idoneidad e interés de los resultados para la comunidad científica; y es en este punto donde es relevante la figura del asesor de tesis, pues la responsabilidad que recae sobre este conlleva a asumir que tanto su experiencia en investigación así como el ideal de que este tenga la experiencia de publicar trabajos o resultados de investigación, hace suponer que podría ser una figura de apoyo importante a la hora de orientar la realización de trabajos con potencial publicable e impacto social. Sin embargo, algunos estudios y opiniones vertidas en la literatura científica respecto a su labor evidencian que este no estaría cumpliendo un trabajo orientado a la producción científica estudiantil, es más, algunos reportes como el de Ticse, Ygreda, y Samalvides (2014) concluyen que la labor del asesor es un factor que influye en la problemática de culminación de las tesis, lo cual también es corroborado por Mejia, Cáceres, Vera, IngaBerrospi, y Mayta-Tristán (2016) quienes encontraron que cerca del 10\% de una población de médicos recién egresados, se mostraron insatisfechos con la labor de su asesor, además, el 25.8\% indicaron un mal desempeño en el apoyo de los trámites administrativos y el $22.7 \%$ refiere que no recibieron algún incentivo u orientación para publicar el trabajo.

Precisamente son estos datos los que generan gran preocupación para el sistema universitario, ya que se espera que la figura del asesor vaya más allá de ser solo un veedor en el proceso de tesis, en cambio, es necesario que este goce de las competencias necesarias para orientar las propuestas de investigación hacia la resolución de problemas sociales y de salud pública del país, así también logre impulsar trabajos que puedan generar resultados publicables y útiles para 
la comunidad científica; sin embargo, este sigue siendo un ideal para el sistema pues los reportes encontrados en la literatura científica, como es el caso de Atamari-Anahui, Sucasaca-Rodríguez, y Marroquin-Santa Cruz (2016) quienes luego de analizar la producción de 68 asesores de una escuela de medicina peruana, revelan que menos de la mitad había publicado alguna vez en su vida, y solo uno de cada 8 lo había hecho en los últimos tres años. Aunque este último estudio, y anteriores citados solo revelan la realidad de las escuelas de medicina, es posible asumir que la misma problemática sea latente en otros contextos como son las escuelas de psicología, pues son muy pocos los estudios que revelan la situación de la producción científica estudiantil (Huaraca, Apaza, Mejia, 2017; Vera-Villaroel, Lopez-Lopez, Lillo, \& Silva, 2011), y aún más escasas las investigaciones que analizan la producción de los asesores de tesis en este campo; por lo tanto, y ante los análisis realizados en este contexto es que surge la necesidad de realizar la presente investigación con el objetivo de describir las características de los asesores de tesis de pregrado en una escuela de psicología y analizar su producción científica en el periodo 2014 - 2017.

\section{Método}

Se realizó un estudio descriptivo-observacional, donde se analizaron la totalidad de las tesis de pregrado de Psicología aprobadas y disponibles en el repositorio digital de la institución en cuestión, correspondientes al periodo 2014 - 2017. Por lo tanto, se observaron 161 trabajos de grado, sin embargo debido a restricciones de los autores solo se pudo analizar 149 en archivo extenso.

En una primera instancia se revisó cada una de las tesis aprobadas, recopilando el nombre del docente consignado como asesor. Para corroborar la información también se recurrió a la página del mismo archivo donde se consigna las firmas del jurado calificador. Terminada la recopilación se identificaron 37 docentes adscritos a la Escuela Académico Profesional de Psicología tanto de la sede principal Lima y dos filiales: Tarapoto y Juliaca.

En una segunda etapa, se recurrió al buscador Google Académico, el cual se caracteriza por ser un motor de búsqueda de contenido especializado respecto a literatura académico-científico, además, es bien sabido que este buscador contiene artículos publicados de todas las revistas científicas electrónicas existentes a nivel internacional (Falagas, Pitsouni, Malietzis, \& Pappas, 2007). Así mismo, la estrategia de búsqueda consistió en redactar los apellidos y nombres completos de los docentes identificados como asesores, de esa manera, se contabilizó el número 
total de artículos donde figuraban como autor principal y co-autores respectivamente. Al respecto, en algunos casos se pudo realizar el seguimiento de sus publicaciones en Google Scholar Citations de donde también se extrajo información relevante.

Respecto al análisis estadístico, se efectuó un análisis descriptivo de los datos en el software SPSS 22.0, para presentarlos utilizando frecuencias y porcentajes.

\section{Resultados}

En la tabla 1 se observan los resultados del análisis descriptivo respecto a las principales características de los asesores de tesis de Psicología de una universidad privada del Perú. En el periodo 2014 - 2017 intervinieron 37 docentes que asumieron la responsabilidad de ser asesores de trabajos de grado como requisito para obtener el título profesional de Psicólogo, de los cuales el $70.3 \%$ fueron docentes mujeres y el $29.7 \%$ docentes varones; respecto al grado académico que ostentan, el $64.9 \%$ cuenta con título profesional, mientras que el $21.6 \%$ evidencia grado de maestro y solo el $13.5 \%$ grado de doctor; también se identificó que el $43.2 \%$ pertenecen a la sede principal ubicada en la ciudad de Lima, el $32.4 \%$ a la sede ubicada en la ciudad de Juliaca y $24.3 \%$ a la sede de Tarapoto.

Respecto a la producción científica de los asesores, se observa que solo el $27 \%$, es decir 10 docentes evidencian haber publicado un artículo científico alguna vez en su vida, mientras que el $73 \%$ no evidencia alguno. En cuanto a aquellos que alguna vez publicaron, el $16.2 \%$ evidencia publicaciones en los últimos 3 años y el $21.6 \%$ dentro de los últimos 5 años. Finalmente, se observa que el $89.1 \%$ de la producción corresponde a artículos originales mientras que solo un 5.4\% a cartas al editor y editoriales respectivamente.

Tabla 1

Características de los asesores de tesis de pregrado. Periodo 2014 - 2017

\begin{tabular}{llrr}
\hline Variables & & $\mathrm{f}$ & $\%$ \\
\hline Sexo & Femenino & 26 & 70.3 \\
& Masculino & 11 & 29.7 \\
\hline Grado académico & Título profesional & 24 & 64.9 \\
& Magister & 8 & 21.6 \\
& Doctor & 5 & 13.5 \\
\hline Sede & Lima & 16 & 43.2 \\
& Tarapoto & 9 & 24.3 \\
& Juliaca & 12 & 32.4 \\
\hline
\end{tabular}




\begin{tabular}{llrr}
\hline Publico alguna vez en la vida & $\mathrm{Si}$ & 10 & 27.0 \\
& $\mathrm{No}$ & 27 & 73.0 \\
\hline Publicó en los últimos 3 años & $\mathrm{Si}$ & 6 & 16.2 \\
& $\mathrm{No}$ & 31 & 83.8 \\
\hline Publicó en los últimos 5 años & $\mathrm{Si}$ & 8 & 21.6 \\
& $\mathrm{No}$ & 33 & 78.4 \\
\hline Tipo de articulo & Original & 2 & 89.1 \\
& Carta al editor & 2 & 5.4 \\
& Editorial & & 5.4 \\
\hline
\end{tabular}

Fuente propia

Seguidamente, en la tabla 2 se puede observar que la producción científica de los asesores que publican (10 docentes) suma un total de 37 artículos, de los cuales, más de la mitad (54.04\%) fueron aceptados y difundidos en dos revistas peruanas como son Apuntes Universitarios (AU) y la Revista Científica de Ciencias de la Salud (RCCS), ambas pertenecientes a la Universidad Peruana Unión; sin embargo de estas, solo una cuenta con indexación que denota alta calidad como es Web of Science y Scielo Perú (AU). Continuando, se observan algunas publicaciones en menor frecuencia en revistas prestigiosas del extranjero como es el caso de la colombiana Duazary (2) indexada en WoS, Comupters in Human Behavior del Reino Unido (1) indexada en SCOPUS, interdisciplinaria de Argentina (1) indexada en WoS, SCOPUS y Scielo, Educación Médica de España (1) indexada en SCOPUS y la Revista Internacional de Ciencias Sociales de Paraguay (1) indexada en Scielo. Finalmente, también se observan publicaciones en otras revistas peruanas de las cuales cabe resaltar aquellas que tienen indexaciones de alta calidad como es el caso de Propósitos y Representaciones de la Universidad San Ignacio de Loyola (1).

Tabla 2

Características de la producción científica de los asesores de tesis de pregrado. Periodo 2014 2017

\begin{tabular}{llccccrr}
\hline & & \multicolumn{3}{c}{} & \multicolumn{3}{c}{$\begin{array}{c}\text { Artículos } \\
\text { publicados }\end{array}$} \\
\cline { 3 - 7 } \multicolumn{1}{c}{ Revista } & \multicolumn{1}{c}{ País } & Scopus & WoS & Scielo & Otros & f & $\%$ \\
\hline Apuntes universitarios & Perún & NO & SI & SI & SI & 10 & 27.0 \\
Científica de Ciencias de la Salud & Perú & NO & NO & NO & SI & 10 & 27.0 \\
Revista de Investigación Universitaria & Perú & NO & NO & NO & SI & 3 & 8.1 \\
Apuntes Psicológicos & Perú & NO & NO & NO & SI & 2 & 5.4 \\
Duazary & Colombia & NO & SI & NO & SI & 2 & 5.4 \\
Computers in Human Behavior & Reino Unido & SI & SI & NO & SI & 2 & 5.4 \\
\hline
\end{tabular}




\begin{tabular}{llcccrrr}
\hline Temática Psicológica & Perú & NO & NO & NO & SI & 2 & 5.4 \\
Interdisciplinaria & Argentina & SI & SI & SI & SI & 1 & 2.7 \\
Educación Medica & España & SI & NO & SI & SI & 1 & 2.7 \\
Internacional de Ciencias Sociales & Paraguay & NO & NO & SI & SI & 1 & 2.7 \\
Muro de la Investigación & Perú & NO & NO & NO & SI & 1 & 2.7 \\
Revista de Psicología & Perú & NO & NO & NO & SI & 1 & 2.7 \\
Propósitos y Representaciones & Perú & NO & SI & SI & SI & 1 & 2.7 \\
Total & & & & & & 37 & 100 \\
\hline
\end{tabular}

Fuente propia

\section{Discusión}

Se halló que en el periodo 2014 - 2017 intervinieron 37 docentes adscritos a la Escuela Profesional de Psicología, quienes asumieron el rol de asesores de tesis conducentes a la obtención del título profesional de Psicólogo. Existen más docentes mujeres que varones y solo 13 (8 magister y 5 doctores) cumplen con el grado académico mínimo (Magister) para asesorar tesis de licenciatura, según el Reglamento General de Investigación de la universidad en cuestión, además, 16 de ellos corresponden al staff de asesores de la sede principal, mientras que 12 son de Juliaca y 9 de Tarapoto. Un primer análisis respecto a estos hallazgos conduce a esclarecer el por qué 24 docentes que aún no tienen el grado requerido fungen como asesores, al respecto, una explicación se encuentra al comprender que estos ejercían la docencia universitaria antes de instaurarse la nueva ley universitaria, por ende, aún se encuentran en el periodo de adecuación establecido según ley; igualmente, el hecho de que existan más asesores en la sede principal que en las filiales obedece a una situación de demanda de alumnos, pues es bien sabido que las sedes principales son aquellas donde se encuentran una mayor cantidad de población estudiantil.

Otro de los hallazgos importantes tiene que ver con la producción científica de los docentes que ejercen asesoría de trabajos de grado. Se encontró que solo 10 docentes de un total de 37 lograron publicar alguna vez en su vida, de los cuales seis aun evidencian contribuciones dentro de los últimos 3 años. Estos resultados son similares, aunque no en su totalidad, a los hallados por Taype-Rondán, Carbajal-Castro, Arrunategui-Salas, y Chambi-Torres (2012) quienes luego de analizar la producción de 67 asesores encontraron que solo 21 había publicado algún artículo original; de igual manera, contrastables al de un estudio reciente realizado por Atamari-Anahui et al. (2016) quienes hallaron que solo 32 de 68 asesores habían publicado alguna vez en su vida, además, al igual que en los resultados de la presente investigación (16.2\% de docentes publicaron en los últimos 3 años) se encontró que solo el 11.8\% mantenía publicaciones en los últimos 3 años. 
En suma, al parecer estos datos son indicadores frecuente que evidencia que en algunas universidades peruanas es frecuente que los tesistas sean asesorados por docentes sin ninguna publicación en los últimos cinco años (Ticse, Pamo, Samalvides, \& Quispe, 2014). Esto, claramente influye de manera negativa la producción científica estudiantil y a la vez haría comprender el porqué de una limitada publicación de tesis en el Perú (J Alarco, Aguirre-cuadros, Aliaga-Chávez, \& Alvarez-Andrade, 2010; Atamari-Anahui, Roque-Roque, Robles-Mendoza, Nina Moreno, \& Falcón-Huancahuiri, 2015b; Taype-Rondán et al., 2012) puesto que si el propio asesor no concibe la publicación como parte de su labor como investigador entonces mucho menos lo hará quien se encuentre en la posición de ser su asesorado.

Otro análisis relevante tiene que ver con la producción científica de aquellos asesores (10 docentes) que evidenciaron artículos publicados en revistas científicas indizadas. Se encontró que el número total de trabajos hallados en la literatura científica a través del buscador de Google Académico fue de 37, de los cuales en su mayoría (54.04\%) fueron publicados en dos revistas científicas que la propia universidad gestiona, cabe resaltar que de estas solo una evidencia indexaciones de alta calidad (WoS y Scielo) como es el caso de Apuntes Universitarios, sin embargo, ninguna de las mencionadas es considerada como revista de especialidad (Scope Temático referente a líneas de investigación en Psicología). Otras publicaciones en cambio, si llegaron a ser divulgadas por revistas de especialidad como es el caso de interdisciplinaria (1), Revista de Psicología (1), Propósitos y Representaciones (1) y Computers in Human Behavior (1). Una explicación ante estos resultados encuentra sentido al reconocer que habría algún desconocimiento por parte de los autores, que en su defecto son pocos, acerca del valor de las publicaciones en revistas de corriente principal, aunado a esto, el hecho de que la tasa de rechazo en revistas prestigiosas es alta.

En conclusión, se asume que la tasa publicación de los asesores de tesis de pregrado en psicología de la universidad en cuestión es baja y preocupante, lo cual evidencia grandes limitaciones respecto a las competencias investigativas en los docentes asesores, además, se revela que una gran parte de los involucrados ostenta aun el título profesional lo cual estaría develando una falta de capacitación y formación en investigación científica. Por lo tanto, estos hallazgos corroboran lo evidenciado en la literatura científica respecto a la problemática de las tesis y las competencias del asesor.

\section{Referencias}


Alarco, J., Aguirre-Cuadros, E., Aliaga-Chávez, Y., \& Alvarez-Andrade, E.V. (2010). Factores asociados a la realización de tesis en pregrado de Medicina en una universidad pública del Perú. Ciencia e Investigación Médica Estudiantil Latinoamericana (CIMEL), 15(2), 66-70. Recuperado de http://www.redalyc.org/pdf/717/71721155005.pdf

Alarco, J., Changllio-Calle, G., \& Cahuana-Salazar, M. (2017). Investigación en pregrado: interés según sexo y ciclo académico. Educacion Medica, 18(1), 67-73. doi: https://doi.org/10.1016/j.edumed.2016.04.004

Atamari-Anahui, N., Roque-Roque, J. S., Robles-Mendoza, R. A., Nina Moreno, P. I., \& FalcónHuancahuiri, B. M. (2015a). Publicación de tesis de pregrado en una facultad de Medicina en Cusco , Perú. Revista Medica Herediana, 26(4), 217-221. doi: https://doi.org/10.20453/rmh.v26i4.2707

Atamari-Anahui, N., Sucasaca-Rodríguez, C., \& Marroquin-Santa Cruz, J. A. (2016). Publicación científica de asesores de tesis de pregrado en una escuela de medicina de Cusco, Perú. Investigación En Educación Médica, 5(20), 279-280. doi: https://doi.org/10.1016/j.riem.2016.05.002

Castro-Rodríguez, Y. (2018). Producción científica estudiantil en revistas odontológicas peruanas durante el periodo 2012 al 2017. Educacion Medica, (xx). doi: https://doi.org/10.1016/j.edumed.2018.02.008

Falagas, M. E., Pitsouni, E. I., Malietzis, G. A., \& Pappas, G. (2007). Comparison of PubMed, Scopus, Web of Science, and Google Scholar: strengths and weaknesses. The FASEB Journal, 22(2), 338-342. doi: https://doi.org/10.1096/fj.07-9492LSF

Huaraca, H., Apaza, C.M., Mejia, A., Alvarez, C. (2017). Realidad peruana de la publicación científica estudiantil en los últimos diez años. Educación Médica Superior, 31(3). Recuperado de http://www.ems.sld.cu/index.php/ems/article/view/1019/624

León, F. (2016). ¿Qué significa una tesis de investigación en psicología en el Perú? Revista Persona, (19), 151-166. Recuperado de http://www.redalyc.org/pdf/658/65821107.pdf

Mejia, C. R., Cáceres, O. J., Vera, C. A., Inga-Berrospi, F., \& Mayta-Tristán, P. (2016). Percepción y factores asociados a insatisfacción que los médicos recién graduados tienen de sus asesores de tesis, Lima-Perú. Revista Cubana de Educacion Medica Superior, 30(4), 340-348. Recuperado de http://scielo.sld.cu/scielo.php?script=sci_arttext\&pid=S0864- 


\section{0}

Ministerio de Educacion. (2014). Ley Universitaria: Ley Nº 30220. Lima: El Peruano.

Recuperado de http://www.minedu.gob.pe/reforma-universitaria/pdf/ley_universitaria.pdf

Miyahira, J. (2015). ¿Por qué la tasa de publicación de las tesis en Medicina es baja?: Posibles explicaciones. Revista Medica Herediana, 26(4), 207-208. Recuperado de http://www.scielo.org.pe/pdf/rmh/v26n4/a01v26n4.pdf

San Martin, F., \& Garcia, M. (2006). La tesis y su problemática en la Facultad de Medicina Veterinaria de la UNMSM. Rev Inv Vet Perú, 17(1), 81-88. Recuperado de http://www.scielo.org.pe/pdf/rivep/v17n1/a15v17n1.pdf

Taype-Rondán, Á., Carbajal-Castro, C., Arrunategui-Salas, G., \& Chambi-Torres, J. (2012). Limitada publicación de tesis de pregrado en una facultad de medicina de Lima, Perú, 20002009. Anales de La Facultad de Medicina, 73(6), 153-157. doi: https://doi.org/10.15381/anales.v73i2.858

Ticse, R., Pamo, O., Samalvides, F., \& Quispe, T. (2014). Factores asociados a la culminación del proyecto de investigación requerido para optar el título de especialista en una universidad peruana. Revista Médica Peruana de Medicina Experimental y Salud Pública, 31(1), 48-55. Recuperada de http://www.scielo.org.pe/pdf/rins/v31n1/a07v31n1.pdf

Ticse, R., Ygreda, P., \& Samalvides, F. (2014). Publicación de los proyectos de investigación para la titulación como Medicos especialistas de una Universidad Peruana, 2007-2010. Revista Peruana de Medicina Experimental y Salud Publica, 31(2), 292-296. doi: https://doi.org/36326085012

Vera-Villaroel, P., Lopez-Lopez, W., Lillo, S., \& Silva, L. M. (2011). La producción científica en psicología latinoamericana : Un análisis de la investigación por países. Revista Latinoamericana de Psicología. 43(1), 95-104. Recuperado de http://www.redalyc.org/articulo.oa?id=80520078009 